\title{
Parásitos intestinales en perros y gatos con dueño de la ciudad de Barranquilla, Colombia
}

\author{
Intestinal parasites in dogs and cats with owners of Barranquilla, Colombia \\ Luz Adriana Sarmiento-Rubiano ${ }^{1,5}$, Lucena Delgado ${ }^{2}$, Julieannie Paola Ruiz \\ María C. Sarmiento ${ }^{4}$, Jimmy Becerra $^{1}$
}

\section{Resumen}

El objetivo del estudio fue evaluar el parasitismo intestinal en perros y gatos con dueño de la ciudad de Barranquilla. Se realizó un estudio descriptivo retrospectivo donde se incluyeron los reportes de análisis coprológicos realizados en un laboratorio clínico veterinario durante los años 2014 y 2015 a 925 perros y 45 gatos provenientes de 26 veterinarias ubicadas en la ciudad. El $73.3 \%$ de los perros y el $62.2 \%$ de los gatos presentaban algún tipo de parásito intestinal, sin diferencia significativa entre especies. Los parásitos más frecuentes fueron los helmintos Toxocara sp $(12.4$ y $8.9 \%$ en perros y gatos, respectivamente), seguido de Ancylostoma sp (3.4 y 4.4\% en perros y gatos, respectivamente). Los protozoarios más frecuentes fueron Entamoeba sp, Isospora sp y Giardia sp en perros y los dos últimos en gatos. La alta frecuencia de parásitos intestinales de transmisión zoonótica encontrados en perros y gatos demuestra la necesidad de instaurar medidas correctivas y preventivas desde el ámbito de la salud pública para su control.

Palabras clave: parásitos intestinales; Giardia; Blastocystis; helmintos; zoonosis; protozoarios

\footnotetext{
${ }^{1}$ Grupo de Investigación Alimentación y Comportamiento Humano, Universidad Metropolitana Barranquilla, Colombia

${ }^{2}$ Universidad Metropolitana de Barranquilla, Colombia

${ }^{3}$ Centro de Diagnóstico Veterinario Especializado BIOVET, Barranquilla, Colombia

${ }^{4}$ Universidad del Sinú, Cartagena, Colombia

${ }^{5}$ E-mail:lusarru@hotmail.com
}

Recibido: 16 de marzo de 2018

Aceptado para publicación: 14 de septiembre de 2018 
The aim of this study was to evaluate the intestinal parasitism in dogs and cats with owners of the city of Barranquilla. A retrospective descriptive study was carried out, which included the reports of coprological analyses made in a veterinary clinical laboratory during the years 2014 and 2015 to 925 dogs and 45 cats from 26 veterinary centres located in the city. Results showed that $73.3 \%$ of dogs and $62.2 \%$ of cats had some type of intestinal parasites, without significant difference between species. The most frequent helminths were Toxocara sp (12.4 and 8.9\% in dogs and cats, respectively), followed by Ancylostoma sp (3.4 and 4.4\% in dogs and cats, respectively). The most frequent protozoa were Entamoeba sp, Isospora sp and Giardia sp in dogs and the last two in cats. The high frequency of intestinal parasites of zoonotic transmission found in dogs and cats showed the need to establish corrective and preventive measures for their control based on public health.

Key words: intestinal parasites; Giardia; Blastocystis; helminths; zoonosis; Protozoa

\section{INTRODUCCIÓN}

De los más de 1400 patógenos humanos conocidos, cerca del $58 \%$ son de origen zoonótico, y el 73\% de los 177 patógenos considerados por la OMS como reemergentes están relacionados al contacto del humano con una fuente animal (Woolhouse y Gowtage-Sequeria, 2005; Jones et al., 2013).

Los protozoarios Giardia sp, Entamoeba histolytica, Cyclospora y Cryptosporidium sp son los parásitos intestinales más comúnmente encontrados en la población mundial y están asociados a desordenes gastrointestinales y diarrea en personas sanas o inmunológicamente comprometidas, siendo las giardiasis y las amebiasis las principales causas de diarrea asociada a parásitos en el mundo (Haque, 2007). Estos parásitos considerados de transmisión zoonótica pueden estar presentes en animales domésticos, como perros y gatos (Sotiriadou et al., 2013; Dantas-Torres y Otranto, 2014). Estas mascotas actúan como potenciales reservorios y son fuente constante de contaminación directa, principalmente para la población infantil con quienes interaccionan constantemente, pero también contaminando indirectamente las fuentes de agua, el suelo y los alimentos a través de sus heces.

Las parasitosis intestinales son consideradas un problema de salud pública mundial, que además de afectar la salud humana, tienen efectos sociales, económicos y culturales asociados con la perpetuación de la pobreza y la desigualdad de los pueblos. Las regiones con mayor prevalencia de parasitosis intestinales son las zonas tropicales y subtropicales como África subsahariana, China, Asia oriental y América Latina, donde aproximadamente 270 millones de niños en edad preescolar y 600 millones en edad escolar están en alto riesgo de infección por estos parásitos (BID et al., 2011).

El gran impacto de las zoonosis en la salud humana hace pertinente y oportuno la realización de estudios que ayuden a comprendery definir los posibles riesgos de transmisión de estas patologías, más aún cuando involucran mascotas como perros y gatos que conviven tan íntimamente con las personas. El objetivo del presente estudio fue evaluar el parasitismo intestinal en perros y gatos con dueño de la ciudad de Barranquilla en 2014 y 2015. 


\section{Materiales y Métodos}

Se realizó un estudio descriptivo retrospectivo en el que se incluyeron los reportes de análisis coprológicos realizados a 925 perros y 45 gatos en el laboratorio clínico veterinario BIOVET de la ciudad de Barranquilla, durante los años 2014 y 2015, provenientes de 26 centros de atención veterinaria de la ciudad de Barranquilla, Colombia. De estos, ocho están ubicados en el sector norte de la ciudad (Riomar) con el aporte de 259 muestras $(26.7 \%)$ (251 de perros y 8 de gatos), diez se ubican en el sector centro (centro histórico) con el aporte de 340 muestras (35.0\%) (325 de perros y 15 de gatos) y las otras ocho en el sector sur (sur occidente y sur oriente) con el aporte de 371 muestras (38.3\%) (349 de perros y 22 de gatos).

Las muestras fecales (10-20 g) fueron aportadas por los dueños de los perros y gatos como examen rutinario de control a sus mascotas. El diagnóstico parasitológico lo realizó una laboratorista especialista en el tema mediante examen directo microscópico de las muestras fecales frescas con solución salina y lugol para la detección de Dipylidium $\mathrm{sp}$, entre otros parásitos sensibles al rápido deterioro. Además, se hizo la técnica de concentración por flotación con solución salina concentrada para la identificación de formas parasitarias flotantes (Figueroa-Castillo et al., 2015).

Para el análisis de los resultados, los perros fueron clasificados en dos categorías: mestizos (animales de ascendencia desconocida con características de dos o más tipos de razas) y de raza (de acuerdo a la clasificación de la Federación Cinológica Internacional - FCI - de la Organización Canina Mundial) (FCI, 2018). Para la designación del tipo racial en el caso de los gatos se consideró la clasificación de la Federación Internacional Felina (FIFe) (FIFe, 2018).
Se realizó un análisis descriptivo exploratorio de los resultados con el fin de establecer la frecuencia absoluta y relativa de los parásitos presentes en las muestras. Se compararon los resultados entre razas de perros, entre perros y gatos, y entre zonas de muestreo mediante la prueba de Chi cuadrado para variables categóricas. Se utilizó el programa estadístico Statgraphics plus.

\section{Resultados}

E1 $89.1 \%(n=824)$ de los perros muestreados pertenecían a alguna de las razas clasificadas en 10 grupos por la FCI y el $10.9 \%(n=101)$ fueron mestizos. En el caso de los gatos, cuatro fueron de raza Persa y los 41 restantes fueron mestizos. El 73.3\% de los perros y el $62.2 \%$ de los gatos presentaban algún tipo de parásito intestinal, aunque sin diferencia significativa entre especies (Cuadro 1).

No se observaron diferencias significativas entre grupos de razas (Cuadro 2), con excepción del Grupo I (Pastor y Boyeros) $(p<0.05)$, aunque hay que hacer notar el re-

Cuadro 1. Parasitismo intestinal en perros y gatos atendidos en clínicas veterinarias de la ciudad de Barranquilla, Colombia (20142015)

\begin{tabular}{lcc}
\hline Animales & $\begin{array}{c}\text { Total } \\
\text { (n) }\end{array}$ & $\begin{array}{c}\text { Parasitado } \\
\%\end{array}$ \\
\hline Perros & & \\
Mestizos & 101 & 75.2 \\
De raza & 824 & 73.1 \\
\hline Total & 925 & 73.3 \\
Gatos & & \\
Mestizos & 41 & 61.0 \\
De raza & 4 & 75.0 \\
\hline Total & 45 & 62.2 \\
\hline
\end{tabular}


Cuadro 2. Parasitismo intestinal en perros de raza, según la clasificación de la Federación Cinológica Internacional (FCI), atendidos en clínicas veterinarias de la ciudad de Barranquilla, Colombia (2014-2015)

\begin{tabular}{lcc}
\hline $\begin{array}{l}\text { Razas de perros según la Federación Cinológica } \\
\text { Internacional (FCI) }\end{array}$ & $\begin{array}{c}\text { Total } \\
(\mathrm{n})\end{array}$ & $\begin{array}{c}\text { Parasitado } \\
\%\end{array}$ \\
\hline I. Pastor y Boyeros & 5 & 40.0 \\
II. Pinscher, Schnauzer, Molosoides y Boyeros Suizos & 200 & 76.0 \\
III. Terriers & 111 & 73.0 \\
V. Spitz y Primitivo & 53 & 77.4 \\
VI. Sabueso y de rastro & 36 & 72.2 \\
VII. De muestra & 6 & 66.7 \\
VIII. De caza, perros de agua & 170 & 71.2 \\
IX. De compañía & 243 & 71.6 \\
\hline Total & 824 & 73.1 \\
\hline
\end{tabular}

Cuadro 3. Frecuencia relativa (\%) de los parásitos intestinales en muestras fecales de perros y gatos atendidos en clínicas veterinarias de la ciudad de Barranquilla, Colombia (2014-2015)

\begin{tabular}{llcccc}
\hline \multirow{2}{*}{ Parásito } & & \multicolumn{3}{c}{ Perros } & \multirow{2}{*}{ Gatos } \\
\cline { 3 - 5 } & & Total & De raza & Mestizo & \\
\hline \multirow{2}{*}{ Nematodos } & Strongylus sp & 1.2 & 1.1 & 2.0 & 0 \\
& Ancylostoma sp & 3.4 & 3.3 & 4.0 & 4.4 \\
& Toxocara canis - T. felis & 12.4 & 12.3 & 13.9 & 8.9 \\
& Uncinaria & 1.7 & 1.7 & 2.0 & 0 \\
Cestodos & Ascaris sp & 0.2 & 0.2 & 0 & 0 \\
Protozoarios & Dipylidium & 0.8 & 0.7 & 1.0 & 0 \\
& Entamoeba sp & 35.8 & 36.2 & 32.7 & 0 \\
& Giardia & 18.1 & 18.3 & 15.8 & 20.0 \\
& Isospora sp & 20.5 & 19.7 & 27.7 & 33.3 \\
& Trichomona & 1.3 & 1.3 & 1.0 & 2.2 \\
& Balantidium & 0.2 & 0.2 & 0 & 0 \\
& Schilomastix & 0.5 & 0.6 & 0 & 0 \\
\hline
\end{tabular}

ducido número de animales en este grupo $(\mathrm{n}=5)$. En el caso de los gatos, el parasitismo fue significativamente mayor en los de raza Persa en comparación con el grupo de mestizos, pero igualmente con un número reducido de gatos muestreados $(n=4)$.
Entre los animales parasitados, la frecuencia de helmintiasis fue mayor en los perros $(25.8 \%)$ que en los gatos (15.5\%) $(\mathrm{p}<0.05)$, y solo siete perros presentaron más de un helminto. Los protozoarios afectaron al $84.8 \%$ de los perros y al $82.1 \%$ de los ga- 
tos parasitados. La presencia de helmintos y protozoarios en una misma muestra fecal se observó en el 10.6\% de los perros y tres gatos tenían infección por dos protozoarios a la vez, siendo en todos los casos Giardia e Isospora.

Toxocara sp fue el nematodo más frecuente encontrado en las heces de los perros (12.4\%) y de los gatos (8.9\%), seguido de Ancylostoma sp (3.4 y 4.4\% en perros y gatos, respectivamente) (Cuadro 3), y sin diferencias significativas entre las dos especies de mascotas. Los protozoarios más frecuentes fueron Entamoeba sp, Isospora sp y Giardia sp en perros y estos dos últimos en gatos (Cuadro 3). La comparación de las frecuencias de cada parásito entre perros y gatos solo mostró diferencias significativas en el caso de Entamoeba sp e Isospora sp.

No se observaron diferencias significativas de frecuencia de perros y gatos parasitados entre las tres zonas de procedencia de las muestras. No obstante, se notó una menor frecuencia de toxocariasis en la zona norte $(7.7 \%)$ que en las zonas centro y sur (12.6 y $15.1 \%$, respectivamente).

\section{Discusión}

La población de perros y gatos en Barranquilla en 2016 era de 82386 y 31003 ejemplares, respectivamente (Ministerio de Salud y Protección Social, 2016) y si se considera que la población humana estimada era de 1223616 personas en Barranquilla para el mismo año de acuerdo al Departamento Nacional de Estadística (DANE, 2007), se puede inferir que existe un perro por cada 15 habitantes y un gato por cada 40 habitantes. En este estudio se encontró que el $73.3 \%$ de los perros y el $62.2 \%$ de los gatos tenidos como mascotas en los hogares y que fueron atendidos en alguna de las veterinarias de la ciudad de Barranquilla en el periodo 2015-2016 presentaban parásitos intestinales que, además de afectar la salud y bienestar de las mascotas, pueden potencialmente afectar la salud humana.
Los parásitos más frecuentes, Toxocara sp y Ancylostoma sp en las muestras en estudio, son causantes en el humano de larvas migratoria cutáneas asociadas a dermatitis, y en los casos más complicados a neumonía eosinofílica, eritema multiforme, Síndrome de Loeffler y neuroretinitis subaguda, entre otras patologías, dependiendo de la ubicación de la larva y del estado general del hospedero (Macpherson, 2013). Protozoarios como Entamoeba sp y Giardia sp con altas frecuencias en los animales evaluados en este estudio, están asociados a desordenes gastrointestinales y diarrea en niños y adultos y coccidias como Isospora sp afectan de manera importante la salud de pacientes inmunosuprimidos (Fletcher et al., 2012).

Aunque los niveles de parasitismo encontrados en este estudio no reflejan la prevalencia de parasitismo en la población general de perros y gatos de la ciudad de Barranquilla, por incluirse solamente animales atendidos en veterinarias en citas de control, excluyendo la población de animales callejeros y los animales con dueño que no asistieron a los servicios veterinarios, se destaca que las frecuencias encontradas son superiores a la de estudios realizados en otras zonas del país. Así, en perros con dueño del área urbana del municipio de la Mesa Cundinamarca se reportó 19.6\% (Alarcón et al., 2015), en la zona urbana de Coyaima (Tolima) fue de 53\% (Giraldo y Gonzalez, 2015) y en el municipio de Envigado (Antioquia) fue de 67.9\% (Caraballo et al., 2007), con una amplia variedad de especies de parásitos en cada uno de ellos. Además, en perros callejeros en la ciudad de Bogotá se reportó $88.6 \%$ de perros con helmintiasis (Solarte et al., 2013), mientras que en Veracruz, México, fue de $98.0 \%$ en perros de un refugio (Alvarado-Esquivel, 2015).

El $62.2 \%$ de parasitismo intestinal encontrado en los gatos de este estudio fue superior al $35.1 \%$ reportado en un estudio europeo que incluyó 1519 gatos atendidos en veterinarias de Austria, Bélgica, Francia, Hungría, Italia, Rumania y España, siendo 
Toxocara cati el helminto más común (19.7\%) y Cystoisospora sp y Giardia sp los protozoarios más frecuentes $(9.7$ y $3.2 \%$, respectivamente) (Beugnet et al., 2014); pero ligeramente inferior al $67 \%$ de parasitismo reportado en 146 gatos capturados en áreas públicas de Cuiabá, Brasil (Ramos et al., 2013). En el presente estudio no se encontraron ooquistes de Toxoplasma en las muestras fecales de los gatos, a pesar de que en Colombia se sabe que la positividad de anticuerpos anti-Toxoplasma en la población general es de $47.7 \%$ y entre el 50 y $60 \%$ en mujeres embarazadas (Juliao et al., 1988; Gómez-Marín et al., 1997).

Los resultados, con relación a los ooquistes de Toxoplasma, son coherentes con lo reportado por otros autores, quienes a pesar de evaluar entornos donde se conoce la presencia de este parásito y su prevalencia en la población, no se encuentran los ooquistes en las heces de gatos mediante la evaluación por microscopía directa (Beugnet et al., 2014; Mohsen y Hossein, 2009). Schares et al. (2008) demostraron que el hallazgo de estructuras identificadas como ooquistes de Toxoplasma sp en heces de gatos solo fue posible en el $0.3 \%$ de las muestras y que de estas, solo el $0.09 \%$ fueron identificadas como Toxoplasma gondii por técnicas moleculares. Esto explica que en una muestra tan pequeña de heces de gato como la de este estudio resulta poco probable el hallazgo de estas estructuras.

La presencia de formas parasitarias infectantes en el suelo provenientes de heces de humanos y animales parasitados en áreas urbanas y rurales ha sido ampliamente demostrada. En el $48.3 \%$ de 83 parques de la ciudad de Temuco, Chile, se encontraron huevos de parásitos, siento Toxocara $\mathrm{sp}$ (12.4\%) el más frecuente (Armstrong et al., 2011). En la ciudad de Tunja, Colombia, el $60.7 \%$ de muestras fecales caninas recogidas en parques de la ciudad y el 100\% de muestras de suelo tenían huevos y larvas de parásitos, principalmente Toxocara sp, Ancylostoma spp, Trichuris sp $\mathrm{y}$
Strongyloides sp (Díaz-Anaya et al., 2015). La viabilidad de los huevos de Toxocara sp en el suelo depende de factores como la temperatura, $\mathrm{pH}$, humedad, etc.; sin embargo, se sabe que son muy resistentes y que pueden permanecer infectantes por 6-12 meses, e incluso hasta varios años a bajas temperaturas (Macpherson, 2013).

Resulta pertinente destacar que la Tercera Encuesta Nacional de Parasitismo Intestinal identificó la presencia de parásitos en el $81 \%$ de los niños (Ministerio de Salud y Protección Social y Universidad de Antioquia, 2015), evidenciando que Colombia es un país endémico y con una alta prevalencia de parasitismo intestinal. Por lo tanto, la alta frecuencia de parásitos intestinales que tienen potencial zoonótico encontrados en perros y gatos que asisten a centros veterinarios de la ciudad de Barranquilla, demuestra la necesidad de realizar estudios para definir el parasitismo en animales callejeros, $y$ de instaurar medidas correctivas y preventivas desde el ámbito de la salud pública, que permitan el control de estos parásitos zoonóticos.

\section{Agradecimientos}

A la Universidad Metropolitana y a BIOVET, centro de diagnóstico veterinario especializado Barranquilla, por sus aportes a la realización de este trabajo.

\section{Literatura Citada}

1. Alarcón ZK, Juyo V, Larrotta J. 2015. Gastrointestinales zoonóticos en caninos con dueño del área urbana del municipio de la mesa. Rev Med Vet Zoot 62: 2036. doi: $10.15446 / \mathrm{rfm} v z . v 62 \mathrm{n} 1.49382$

2. Armstrong WA, Oberg C, Orellana JJ. 2011. Presencia de huevos de parásitos con potencial zoonótico en parques y plazas públicas de la ciudad de Temuco, Región de La Araucanía, Chile. Arch Med Vet 3: 127-134. doi: 10.4067/S0301732X2011000200005 
3. Alvarado-Esquivel C, Romero-Salas D, Aguilar-Domínguez M, Cruz-Romero A, Ibarra-Priego N, Pérez-deLeón AA. 2015. Epidemiological assessment of intestinal parasitic infections in dogs at animal shelter in Veracruz, Mexico. Asian Pac J Trop Dis 5: 34-39. doi: 10.1016/S2221-1691(15)30167-2

4. Beugnet F, Bourdeau P, ChalvetMonfray K, Cozma V, Farkas R, Guillot J, Halos L, et al. 2014. Parasites of domestic owned cats in Europe: co-infestations and risk factors. Parasite Vector 7: 1-13. doi: 10.1186/ 1756-3305-7-291

5. [BID] Banco Interamericano de Desarrollo, [OPS] Organización Panamericana de la Salud, Instituto de Vacunas Sabin. 2011. Un llamado a la acción: hacer frente a helmintos transmitidos por el suelo en América Latina y el Caribe. [Internet]. Disponible en: https://www.paho.org/hq/dmdocuments/ 2011/lac-report-esp-final-3-2011.pdf

6. Caraballo A, Jaramillo A, Loaiza J. 2007. Prevalencia de parásitos intestinales en caninos atendidos en el Centro de Veterinaria y Zootecnia de la Universidad CES. Revista CES 2: 24-31.

7. Dantas-Torres F, Otranto D. 2014. Dogs, cats, parasites, and humans in Brazil: opening the black box. Parasite Vector 7: 1-25. doi: 10.1186/1756-3305-7-22

8. [DANE] Departamento Nacional de Estadística. 2007. Proyecciones de población 2005-2020. [Internet]. Disponible en: https://www.dane.gov.co/files/investigaciones/poblacion/proyepobla06_20/8Tablasvida1985_2020.pdf

9. Díaz-Anaya AM, Pulido-Medellín MO, Giraldo-Forero JC. 2015. Nematodos con potencial zoonótico en parques públicos de la ciudad de Tunja, Colombia. Salud Púb Mex 57: 170-176.

10. [FCI] Federación Cinológica Internacional. 2018. Razas reconocidas a título definitivo. [Internet]. Disponible en: http://www.fci.be/es/Nomenclature/
11. [FIFe] Federación Internacional Felina. 2018. Breed standards.[Internet]. Disponible en: http:// fifeweb.org/wp/breeds/breeds_prf_stn.php

12. Figueroa-Castillo JA. Jasso-Villazul C, Liébano-Hernández E, MartínezLabat P, Rodríguez-Vivas RI, ZárateRamos JJ. 2015. Examen coproparasitoscópico. En: Técnicas para el diagnóstico de parásitos con importancia en salud pública y veterinaria. México: AMPAVE-CONASA. p 78-128.

13. Fletcher SM, Stark D, Harkness J, Ellis J. 2012. Enteric protozoa in the developed world: a public health perspective. Clin Microbiol Rev 25: 420449. doi: 10.1128/CMR.05038-11

14. Giraldo JC, Gonzalez AC. 2015. Prevalencia de parásitos intestinales zoonóticos en caninos (Canis lupus familiaris) del área urbana del municipio de coyaima (Tolima). Revista Med 23: $24-34$.

15. Gómez-Marín JE, Montoya-deLondoño MT, Castaño-Osorio JC. 1997. A maternal screening program for congenital toxoplasmosis in Quindío, Colombia and application of mathematical models to estimate incidences using age-stratified data. Am J Trop Med Hyg 57: 180-186.

16. Haque R. 2007. Human intestinal parasites. J Health Popul Nutr 25: 387-391.

17. Jones BA, Grace D, Kock R, Alonso S, Rushton J, Said MY, McKeever D, et al. 2013. Zoonosis emergence linked to agricultural intensification and environmental change. P Natl Acad Sci USA 110: 8399-8404. doi: 10.1073/ pnas. 1208059110

18. Juliao CA, Corredor A, Moreno GS. 1988. Estudio nacional de salud: toxoplasmosis en Colombia. Bogotá: Ministerio de Salud. 67 p.

19. Macpherson NL. 2013. The epidemiology and public health importance of toxocariasis: a zoonosis of global importance. Int J Parasitol 43: 999-1008. doi: 10.1016/j.ijpara.2013.07.004 
20. Ministerio de Salud y Protección Social, Universidad de Antioquia. 2015. [Internet]. Disponible en: https:// www.minsalud.gov.co/sites/rid/Lists/ BibliotecaDigital/RIDE/VS/PP/ET/encuesta-nacional-de-parasitismo-20122014.pdf

21. Ministerio de Salud y Protección social. 2016. Cobertura de vacunación antirrábica de perros y gatos por municipio. [Internet]. Disponible en: https:// www.minsalud.gov.co/sites/rid/Lists/ BibliotecaDigital/RIDE/VS/PP/SA/cobertura-antirrabica-municipal-bimestre12016.pdf

22. Mohsen A, Hossein H. 2009. Gastrointestinal parasites of stray cats in Kashan, Iran. Trop Biomed 26: 16-22.

23. Ramos DG, Alves da Cruz Scheremeta RG, Oliveira AC, Lodovico A, de Campos R. 2013. Survey of helminth parasites of cats from the metropolitan area of Cuiabá, Mato Grosso, Brazil. Rev
Bras Parasitol V 22: 201-206. doi: 10.1590/S1984-29612013000200040

24. Schares G, Vrhovec MG Pantchev N, Herrmann DC, Conraths FJ. 2008. Occurrence of Toxoplasma gondii and Hammondia occysts in the faeces of cats from Germany and other Euopean countries. Vet Parasitol 152: 34-45. doi: 10.1016/j.vetpar.2007.12.004

25. Solarte LD, Castañeda R, Pulido AP. 2013. Gastrointestinal parasites in street dogs in animal shelter from the Bogota D.C, Colombia. Neotrop Helminthol 7: 83-93.

26. Sotiriadou I, Pantchev N, Gassmann D, Karanis P. 2013. Molecular identification of Giardia and Cryptosporidium from dogs and cats. Parasite 20: 8. doi: 10.1051/parasite/201300

27. Woolhouse MEJ, Gowtage-Sequeria S. 2005. Host range and emerging and reemerging pathogens. Emerg Infect Dis 11: 1842-1847. doi: 10.3201/eid1112.050997 